\title{
THE JENSEN AND HERMITE-HADAMARD INEQUALITY ON THE TRIANGLE
}

\begin{abstract}
ZLATKO PAVIĆ
Abstract. We study the functional forms of the most important inequalities concerning convex functions on the triangle. Our intension is to construct the functional form which implies the integral and discrete form of the Jensen inequality, the Fejér, and so the Hermite-Hadamard inequality. To reach this goal, we combine features of positive linear functionals and convex functions.
\end{abstract}

Mathematics subject classification (2010): 26D15, 26B25, 47B38.

Keywords and phrases: Convex function, triangle, positive linear functional.

\section{REFERENCES}

[1] B. D. MacCluer, Elementary Functional Analysis, Springer Science+Business Media, New York, 2009.

[2] L. FeJÉR, Über die Fourierreihen II, Math. Naturwiss. Anz. Ungar. Akad. Wiss., 24 (1906), 369-390.

[3] J. HADAMARD, Étude sur les propriétés des fonctions entières et en particulier d'une fonction considerée par Riemann, J. Math. Pures Appl., 58 (1893), 171-215.

[4] Ch. Hermite, Sur deux limites d'une intégrale définie, Mathesis, 3 (1883), 82.

[5] J. L. W. V. Jensen, Om konvekse Funktioner og Uligheder mellem Middelvardier, Nyt Tidsskr. Math. B, 16 (1905), 49-68.

[6] J. L. W. V. JENSEN, Sur les fonctions convexes et les inégalités entre les valeurs moyennes, Acta Math., 30 (1906), 175-193.

[7] B. JESSEN, Bemarkninger om konvekse Funktioner og Uligheder imellem Middelvardier I, Matematisk Tidsskrift B, (1931), 17-28.

[8] B. JESSEN, Bemarkninger om konvekse Funktioner og Uligheder imellem Middelvardier II, Matematisk Tidsskrift B, (1931), 84-95.

[9] D. LeVermore, Riemann Integrals and Integrability, http://www.terpconnect.umd.edu/ ｌvrmr/2010-2011-F/Classes/MATH410/NOTES/Riemann.pdf, 2010.

[10] Z. Pavić, Functions like convex functions, J. Funct. Spaces, 2015 (2015), Article ID 919470.

[11] Z. PAVIĆ, Inequalities on the triangle, J. Math. Res. Appl., 36 (2016), 51-60.

[12] Z. PaVić, V. Novoselac AND V. PaVIĆ, Functional form of the Jensen and the Hermite-Hadamard inequality, Applied Mathematical Sciences, 9 (2015), 149-159.

[13] T. RAJBA, On the Ohlin lemma for Hermite-Hadamard-Fejér type inequalities, Math. Inequal. Appl., 17 (2014), 557-571.

[14] E. J. McShane, Jensen's inequality, Bull. Amer. Math. Soc., 43 (1937), 521-527. 